\title{
DISCOVERY OF X-RAY EMISSION FROM THE RADIO SNR
}

G352.7-0.1

\author{
K. KINUGASA, K. TORII, AND H. TSUNEMI \\ Graduate School of Science, Osaka University \\ 1-1 Machikaneyama-cho, Toyonaka, Osaka 560, Japan \\ S. YAMAUCHI \\ Faculty of Humanities and Social Sciences, Iwate University \\ K. KOYAMA \\ Graduate School of Science, Kyoto University \\ AND \\ T. DOTANI \\ Institute of Space and Astronautical Science
}

\section{Introduction}

One major objective of our ASCA Galactic Plane Survey Project (AGPSP) is, utilizing the wide and high energy band (up to $10 \mathrm{keV}$ ) X-ray imaging capability and the high spectral resolving power of ASCA, to search possible $\mathrm{X}$-ray SNRs in the Galactic inner disk. The observation of the field including G352.7-0.1 reported in this paper, was performed on 1996 March 14 during the first AO4 survey. We report on the X-ray SNR G352.7-0.1 found in AGPSP. G352.7-0.1 is one of the radio SNRs (Green 1996), and is classified as a shell-like SNR with the size of $8^{\prime} \times 6^{\prime}$.

\section{Analysis and Results}

At X-ray image, we clearly found an extended source with the size of $6^{\prime}$. The center coordinate $(\mathrm{J} 2000)$ of the source is $(\alpha, \delta) \sim\left(17^{h} 27^{m} 42^{s}\right.$, $\left.-35^{\circ} 07^{\prime} 20^{\prime \prime}\right)$. This position is consistent with that of the radio SNR G352.70.1 . From the positional coincidence and the extended structure, we firmly conclude that the X-rays are attributable to the radio SNR. It is the first detection of X-rays from this SNR. We then overlaid SIS image on the VLA 
radio contours at $1465 \mathrm{MHz}$ (Dubner et al. 1993) in figure 1. The SIS image exhibits a shell-like structure which roughly coincides with the radio shell.

The spectra show two remarkable spectral features. One is a low energy turn-off which implies a large interstellar absorption, and the other is the existence of prominent emission lines which correspond to the $\mathrm{K} \alpha$ lines from He-like $\mathrm{Si}, \mathrm{S}$, and Ar. We fitted a non-equilibrium ionization (NEI) model (Masai 1984). The final results we obtained are in table 1. The observed and intrinsic fluxes in the $1-10 \mathrm{keV}$ band are respectively estimated to be $\sim 3.0 \times 10^{-12} \mathrm{erg} \mathrm{cm}{ }^{-2} \mathrm{~s}^{-1}$ and $\sim 1.2 \times 10^{-11} \mathrm{erg} \mathrm{cm}^{-2} \mathrm{~s}^{-1}$.

\section{Discussion}

The observed $N_{\mathrm{H}}$ to G352.7-0.1 is Table 1. The best-fit pathe same to that of the hard com- rameters by the NEI model. ponent of the Galactic ridge emission, hence we reasonably assume that the distance to G352.7-0.1 is nearly equal to that to the Galactic Center $(8.5 \mathrm{kpc})$. Then applying Sedov solution, we can estimate the age and the SN explosion energy to be $t_{\text {age }} \sim 2200 \mathrm{yr}$ and $E_{\mathrm{SN}} \sim 2 \times 10^{50}$ erg, respectively. Taking into account the Galactic abundance gradient, the $\mathrm{S}$ abundance of G352.7-0.1 is consistent with the $S$ abundance near the $\mathrm{GC}$ region.

\begin{tabular}{ll}
\hline Parameters & Values \\
\hline$k T(\mathrm{keV})$ & $2.0(1.3-3.6)$ \\
$\log \tau\left(\mathrm{cm}^{-3} \mathrm{~s}\right)$ & $11.0(10.7-11.5)$ \\
$\mathrm{Si}^{*}$ & $3.7(2.7-5.1)$ \\
$\mathrm{S}^{*}$ & $3.4(2.3-5.0)$ \\
$\mathrm{EM} / 4 \pi \mathrm{D}^{2}\left(\mathrm{~cm}^{-5}\right)$ & $3(2-6) \times 10^{11}$ \\
\hline$N_{\mathrm{H}}\left(10^{22} \mathrm{~cm}^{-2}\right)$ & $2.9(2.4-3.5)$ \\
\hline$\chi^{2} /$ d.o.f & $106.0 / 82$ \\
\hline
\end{tabular}

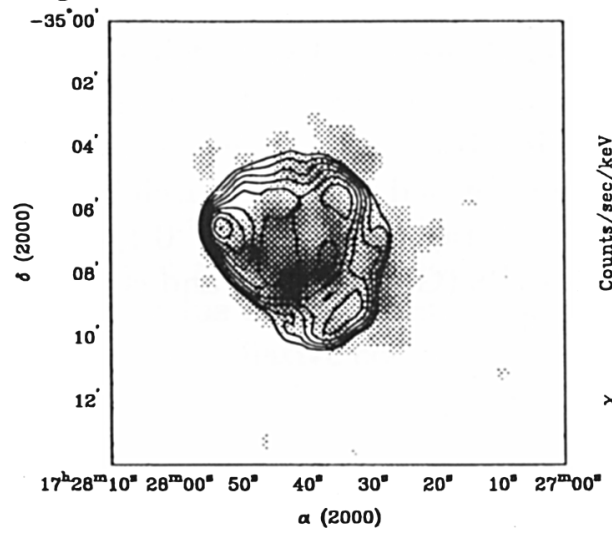

${ }^{*}$ Relative to solar abundance.

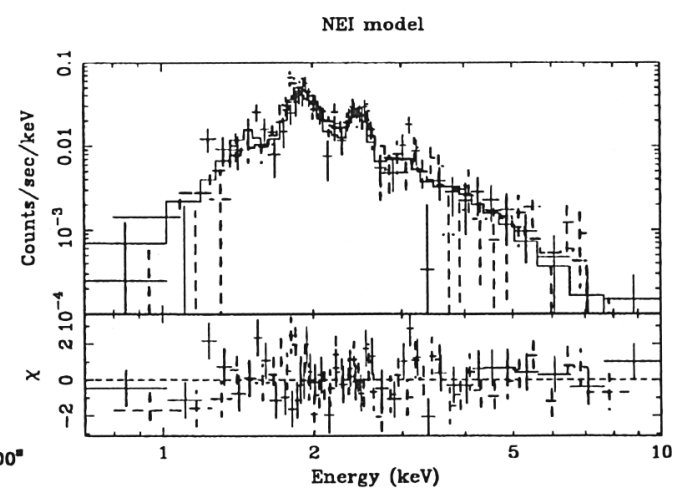

Figure 1. The SIS image (0.7-10keV) of G352.7-0.1 overlaid with the VLA radio contours at $1465 \mathrm{MHz}$.
Figure 2. The energy spectra of G352.7-0.1 and the best-fit NEI model. Solid lines and dashed lines are those of SIS and GIS, respectively. 